\title{
Self-government of complex reading and writing brains informed by cingulo-opercular network for adaptive control and working memory components for language learning
}

\author{
Todd L Richards ${ }^{1 *}$, Robert D Abbott ${ }^{2}$, Kevin Yagle ${ }^{1}$, Dan Peterson ${ }^{1}$, Wendy Raskind ${ }^{3,4}$ and Virginia W Berninger ${ }^{5}$ \\ ${ }^{1}$ Integrated Brain Imaging Center, Department of Radiology, University of Washington, Seattle, USA \\ ${ }^{2}$ Educational Statistics and Measurement, University of Washington, Seattle, USA \\ ${ }^{3}$ Medical Genetics, University of Washington, USA \\ ${ }^{4}$ Psychiatry and Behavioral Sciences, University of Washington, USA \\ ${ }^{5}$ Educational Psychology, Learning Sciences and Human Development, University of Washington, Seattle, USA
}

\begin{abstract}
To understand mental self-government of the developing reading and writing brain, correlations of clustering coefficients on fMRI reading or writing tasks with BASC 2 Adaptivity ratings (time 1 only) or working memory components (time 1 before and time 2 after instruction previously shown to improve achievement and change magnitude of fMRI connectivity) were investigated in 39 students in grades 4 to 9 who varied along a continuum of reading and writing skills. A Philips $3 \mathrm{~T}$ scanner measured connectivity during six leveled fMRI reading tasks (subword-letters and sounds, word-word-specific spellings or affixed words, syntax comprehension — with and without homonym foils or with and without affix foils, and text comprehension) and three fMRI writing tasks- writing next letter in alphabet, adding missing letter in word spelling, and planning for composing. The Brain Connectivity Toolbox generated clustering coefficients based on the cinguloopercular (CO) network; after controlling for multiple comparisons and movement, significant fMRI connectivity clustering coefficients for CO were identified in 8 brain regions bilaterally (cingulate gyrus, superior frontal gyrus, middle frontal gyrus, inferior frontal gyrus, superior temporal gyrus, insula, cingulum-cingulate gyrus, and cingulum-hippocampus). BASC2 Parent Ratings for Adaptivity were correlated with CO clustering coefficients on three reading tasks (letter-sound, word affix judgments and sentence comprehension) and one writing task (writing next letter in alphabet). Before instruction, each behavioral working memory measure (phonology, orthography, morphology, and syntax coding, phonological and orthographic loops for integrating internal language and output codes, and supervisory focused and switching attention) correlated significantly with at least one CO clustering coefficient. After instruction, the patterning of correlations changed with new correlations emerging. Results show that the reading and writing brain's mental government, supported by both CO Adaptive Control and multiple working memory components, had changed in response to instruction during middle childhood/early adolescence.
\end{abstract}

\section{Introduction}

The current study is part of programmatic, interdisciplinary research on the multiple levels of language in the reading brain as well as the transcription (handwriting and spelling) and translation processes (for conversion of thought into written language) in the writing brain. The current study drew upon this prior brain research based on (a) fMRI functional connectivity across the escalating, cascading levels of language (subword, word, syntax, and text) of the reading brain and the transcription and translation processes in and out of the scanner of the writing brain; and (b) fMRI clustering coefficients in the reading brain and the writing brain, informed by graph theory and prior research on the cingulo-opercular (CO) network regarding adaptive control in learning, both before and after instructional intervention. The current study also drew upon assessment studies and family genetics research on developing learners with and without written language learning disabilities that validated behavioral measures of the multiple components of working memory supporting learning to read and write. This grounding in prior research and rationale for extending that research in the current study is explained in the text that follows.

\section{Levels of language in the reading brain}

Much prior research on the reading brain analyzed BOLD response in a region of interest (ROI). However, increasingly fMRI connectivity is being employed from seeds (RO1's) of interest with other brain regions. See [1] for a meta-analysis of seeds for written words that identified left occipital temporal gyrus, left supramarginal gyrus, and left inferior frontal gyrus. See [2] for a seed in the rich club of the human connectome, the left precuneus. An fMRI connectivity study examined connectivity from these four seeds for contrasting levels (units) of language because prior assessment research in developing readers identified intraindividual and interindividual differences in

Correspondence to: Todd L Richards, Integrated Brain Imaging Center, Department of Radiology, University of Washington, Seattle, E-mail: toddr@uw.edu

Key words: Brain's Self Government, Graph Theory Clustering Coefficients, Cingulo-Opercular Network, Adaptive Control in Language Learning, Working Memory Components in Language Learning

Received: July 01, 2017; Accepted: July 28, 2017; Published: July 31, 2017 
Richards TL (2017) Self-government of complex reading and writing brains informed by cingulo-opercular network for adaptive control and working memory components for language learning

levels of language in reading [3,4]. In this fMRI connectivity study, two sets of leveled language tasks for reading (Set 1 subword graphemephoneme, word-specific spelling, syntax with and without homonym foils related to word-specific spellings, and multi-syntax text; Set 2 subword grapheme-phoneme correspondence, word with or without true affixes, syntax with and without affix foils, and multi-syntax text) were analyzed. Common and unique fMRI connectivity from the four seeds (left occipital temporal gyrus, left supramarginal gyrus, left inferior frontal gyrus, and left precuneus) were identified across adjacent levels of language in both Sets 1 and 2 of the reading brains of typical language learners and students with specific learning disabilities in reading during middle childhood and early adolescence; but the unique connectivity was not the same for each level of language [5].

Considering the complexity of such a multi-leveled reading brain and the emerging research showing the complexity of the human connectome [6], the next step in the programmatic research was to apply complex network analysis based on graph theory to identify clustering coefficients within the reading brain. To apply complex network analysis/graph theory analysis, as described in Rubinov and Sporns [7], graphs were constructed for the whole brain, and then clustering coefficients of nodes belonging to a specific functional network referred to as the cingulo-opercular (CO) network were extracted for further analysis. A well-established literature indicates that a CO system of bilateral cingulate, inferior frontal, and anterior insula regions monitors performance and evaluates implementing cognitive control for learning including language learning, for example, aural word recognition [8]. This approach to analyzing the highly interconnected reading brain based on previously identified clustering coefficients at time 1 before instructional intervention and time 2 after instructional intervention showed the reading brain's response to instruction (RTI) that involved changes in patterns of connectivity [9].

\section{Transcription and translation processes of the writing brain}

Likewise, research on the writing brain is transitioning from use of only fMRI BOLD studies of RO1's associated with specific writing or writing-related functions to include fMRI connectivity studies as well. Both transcription (handwriting and spelling) and translation (e.g., planning to express thoughts in written language), which cognitive writing research has shown are important in writing acquisition [3], have been studied in brain imaging studies [10]. For example, Wallis et al. (2017) studied the fMRI connectivity for transcription and translation during scanning and its relationships to the coded transcription and translation during written composing after scanning [11].

Again, given the complexity of the writing brain and the emerging human connectome paradigm, complex network analysis/graph theory analysis, as described in Rubinov and Sporns [7], has also been applied to study the writing brain during middle childhood and early adolescence in students with and without specific learning disabilities. Writing brain graphs were constructed for the whole brain, and then clustering coefficients of nodes belonging to the cinguloopercular (CO) network were extracted for further analysis before and after instructional intervention. The clustering coefficients at time 1 before instructional intervention changed at time 2 after instructional intervention, providing evidence for writing brain's RTI [15].

\section{Extending prior research on complex reading brain and complex writing brain}

The current study was designed to extend the prior research on the complex reading brain and complex writing brain in two ways. To begin with, of interest was whether the parent ratings for Adaptivity on the BASC 2 Behavior Assessment System for Children [12] were correlated with the clustering coefficients related to the $\mathrm{CO}$ network for adaptive control. The BASC 2 Adaptivity Parent Ratings have been validated for their relationships to scholastic achievement in prior studies [12] and for students with and without specific learning disabilities during middle childhood and early adolescence [13]. Also a five-year longitudinal study has shown that parent reports about their children's reading and writing at home are related to their reading and writing achievement at school [14]. If the parent ratings of Adaptivity collected at Time 1 prior to instructional intervention were correlated with clustering coefficients of significant magnitude, then that would lend additional validity to the claim that the $\mathrm{CO}$ network is involved in adaptive control for language learning.

In addition, of interest for the current study was not whether clustering coefficients in the functional networks of the reading brain and the writing brain had changed in response to instruction, which had already been studied $[9,15]$, but rather how the mental selfgovernment for self-regulation of the complex reading brain and the complex writing brain may have changed in response to instruction. Given the complexity of the reading brain and the writing brain and the complexity of the human connectome in general, of interest was how the brain manages this complexity both before and after instruction aimed at the multiple levels of language in the reading brain and the transcription and translation processes of the writing brain $[16,17]$. Of interest was which specific behavioral measures of executive functions for self-regulation would be related to significant clustering coefficients at time 1 and which behavioral measures of executive functions for selfregulation would be related to significant clustering coefficients at time 2. At issue was whether the significant correlations would be the same at time 1 and time 2 and not whether they would change significantly in magnitude from time 1 to time 2 .

On the one hand, clustering coefficients for the nodes in the CO network [8] were of interest because the CO network is one of the reproducible functionally connected resting-state networks and is thought to be involved in cognitive control, that is, detecting errors during task performance [18], thereby signaling possible need for cognitive strategy adjustment [19]. The strength of the within-network CO connectivity predicts cognitive performance [20-22] of tasks involving top-down control [23], facilitating the maintenance of taskrelevant goals, and adjusting behavior based on error information [24].

On the other hand, also of interest was whether the clinical behavioral measures of working memory components validated in prior interdisciplinary research (family genetics and brain imaging) were significantly correlated with clustering coefficients for the fMRI reading tasks and fMRI writing tasks. Changes in these behavior-brain correlations for levels of language in reading and for transcription and translation in writing from before to after instruction would have implications for how the self-government of the complex reading brain system and the complex writing brain system might change in response to instruction. Chomsky (1965) [25] called attention to the role of syntax in language learning, but more recent research has identified other components in addition to syntax coding of accumulating words in working memory supporting language learning: phonology, orthography, and morphology coding (storing and processing words), phonological loop and orthographic loop for integrating internal language codes with motor output codes (mouth or hand, respectively), and attention/executive functions for focusing attention and switching attention $[4,26]$. The phonology, orthography, and morphology components are relevant to learning to read and spell English morphophonemic orthography $[27,28]$. 
Richards TL (2017) Self-government of complex reading and writing brains informed by cingulo-opercular network for adaptive control and working memory components for language learning

\section{Research aims and hypotheses}

Research Aim 1: The first aim was to evaluate correlations between the parent ratings of adaptivity (given only at time 1, before the instructional intervention) and the clustering coefficients in the $\mathrm{CO}$ network. The hypothesis was tested that there would be significant correlations because that network is involved in adaptive control of language learning.

Research Aim 2: The second aim was to evaluate both before (time 1) and after (time 2) instructional intervention the significance of correlations between the working memory measures and the fMRI clustering coefficients, which had first been shown to be of statistically significant magnitude. Results were used to test two hypotheses. The first hypothesis was that before instruction, correlations would be significant between each of the behavioral measures of working memory involved in language learning, and at least one clustering coefficient within the CO Network. The second hypothesis was that some of the correlations at time 1 would not be observed at time 2 or correlations not observed at time 1 would emerge at time 2 because the mental self-government of the language learning brain can change in response to instruction.

\section{Methods}

\section{Study design}

At time 1 before instructional intervention, all students, who were in grades 4 to 9 , completed an assessment battery for oral and written language achievement and component working memory skills for language learning while parents completed BASC 2 parent ratings of their child's Adaptivity [12]. At time 2 after the instructional intervention, students completed the assessment battery with working memory measures again, but parents did not complete BASC 2 Adaptivity ratings again. Both at time 1 and time 2, students completed fMRI functional imaging. One unique feature of the instructional intervention was teaching all levels of language close in time to create functional reading systems and writing systems: subword letter production and subword and word-level phonological, orthographic, and morphological awareness; word reading/decoding and word spelling/encoding; syntax comprehension and syntax construction [17]. Another unique feature was teaching transcription skills (handwriting and spelling) and strategies for generating the next sentence and the evolving multi-sentence text close in time [16,29].

\section{Participants}

Altogether 42 upper elementary and middle school participants (22 males and 20 females; grades 4 to 9; average age 11 years 10 months) participated in brain imaging before and after they completed 18 lessons of specialized writing and reading instruction. Ethnicity was primarily European American (80.5\%), but also Asian American (4.9\%), and other/mixed (14.6\%). Parents' level of education ranged from less than high school (mothers, $0 \%$; fathers $2.4 \%$ ), to high school (2.4\%, mothers; $2.4 \%$ fathers), to more than high school, less than college $(7.1 \%$, mothers; $9.5 \%$, father), to college $(42.9 \%$, mothers, $33.3 \%$, fathers), to more than college ( $47 \%$, mothers; $45 \%$, fathers).

\section{Methods}

\section{Structural and functional brain scanning procedures}

All scans were acquired at the Diagnostic Imaging Sciences Center in collaboration with the Integrated Brain Imaging Center and had
Institutional Review Board approval. Functional magnetic resonance imaging (fMRI) connectivity scans were obtained on a Philips 3T Achieva scanner (release 3.2.2 with the 32-channel head coil). Participants practiced lying still before entering the scanner and were instructed to lie still throughout the scanning. They also practiced the tasks before scanning and were required to achieve $90 \%$ accuracy to continue participation to ensure that the brain imaging results did not reflect inability to do a task.

Each participant was screened for MRI safety before entering the scanner. Physiological monitoring was performed using the Philips pulse oximeter placed on an index finger for cardiac recording, and respiration was recorded using the Philips bellows system where the air-filled bellows pad was placed on the abdomen. Headimmobilization was aided by using an inflatable head-stabilization system (Crania, Elekta).

The following MRI series were acquired before the reading tasks:

1) 3-plane scout view with gradient echo pulse sequence: $T R / T E$ 9.8/4.6 ms; Field of view $250 \times 250 \times 50 \mathrm{~mm}$; acquisition time $30.3 \mathrm{~s}$; 2) reference scan (used in parallel imaging) with gradient echo pulse sequence: TR/TE $4.0 / 0.75 \mathrm{~ms}$; Field of View $530 \times 530 \times 300 \mathrm{~mm}$; acquisition time $44.4 \mathrm{~s} ; 3)$ resting state fMRI scan with echo-planar gradient echo pulse sequence (single shot): TR/TE 2000/25 ms; Field of view $240 \times 240 \times 99 \mathrm{~mm}$; slice orientation transverse, acquisition voxel size $3.0 \times 3.08 \times 3.0 \mathrm{~mm}$; acquisition matrix $80 \times 80 \times 33$; slice thickness 3.0, SENSE factor in the AP direction 2.3; epi factor 37; bandwidth in the EPI frequency direction $1933 \mathrm{~Hz}$, SoftTone factor 3.5, sound pressure $6.1 \mathrm{~dB}, 180$ dynamic scans; 5 dummy scans; fold-over direction AP, acquisition time $6: 14 \mathrm{~min} / \mathrm{s}$; 4) B0 field map imaging with gradient echo pulse sequence and 2 echoes: TR/TE 11/6.3 ms; delta TE $1.0 \mathrm{~ms}$; slice orientation transverse, Field of view $240 \times 240 \times$ $129 \mathrm{~mm}$; voxel size $1.5 \times 1.5 \times 3.0 \mathrm{~mm}$; acquisition matrix $160 \times 160 \times$ 43 , output image magnitude and phase, acquisition time $2: 29 \mathrm{~min} / \mathrm{s} ; \mathbf{5}$ ) MPRAGE structural scan: TR/TE 7.7/3.5 ms, Field of view $256 \times 256$ $\times 176 \mathrm{~mm}$, slice orientation sagittal, voxel size $1 \times 1 \times 1 \mathrm{~mm}$, inversion pulse delay $1100 \mathrm{~ms}$, Sense factor 2 in the AP direction, acquisition time $5: 33 \mathrm{~min} / \mathrm{s}$. 6) fMR during the reading tasks: same parameters as with Resting State fMRI described above except with dynamic scans 396, acquisition time $13.26 \mathrm{~min} / \mathrm{s}$; and 7) fMRI duing the writing tasks: same parameters as with Resting State fMRI described above except with dynamic scans 387, acquisition time $13.08 \mathrm{~min} / \mathrm{s}$. First the reading $\mathrm{fMRI}$ tasks and then the writingfMRI tasks were completed as described next.

\section{fMRI reading tasks}

Unlike an on-off paradigm typically used in fMRI BOLD studies in which a target condition is compared to a resting state or control condition, the current study employed a cascading levels of language paradigm, which has ecological validity for real world reading in which the reader's mind/brain has to process language levels (units) of increasing size from subword to word to sentence/syntax to multisentence text to read a passage. Two sets of tasks each cascading in these levels (units) of language were included that contrast in properties of single words and sentences with or without word foils: Set 1 Tasks 1 , 2, 3, and 6; Set 2, Tasks 1, 4, 5, and 6. The task was always to press a yes or no button to render a judgment about the stimulus presented in each condition. Participants were instructed to look at a fixation cross (no reading task, resting condition; 180 timepoints) or to complete a specific reading task. Because each participant had to achieve $90 \%$ 
Richards TL (2017) Self-government of complex reading and writing brains informed by cingulo-opercular network for adaptive control and working memory components for language learning

accuracy on each task before entering the scanner and it is known that it is difficult for students with SLDs to pay attention to language over time [30], to ensure continuous cognitive engagement, each reading task was presented with self-paced advancing of stimuli (for two minutes; 960 timepoints).

Task 1 subword grapheme-phoneme judgments. Each pair is constructed from a single letter or letter group. The participant was instructed to think about the small sounds that could go with a pair of single letters, a pair of a single letter and a letter group, or a pair of letter groups and then press yes if each letter and/or letter group in that pair presented on the screen can stand for the same sound or no if cannot stand for the same sound. Example of yes pair is " $\mathrm{c}$ " and " $\mathrm{s}$ ". Example of no pair is "d" and " $\mathrm{m}$ ".

Task 2 lexical judgments about correct spellings. The participant was instructed to press yes if the written word on the screen was a correctly spelled real word, but press no if the written word on the screen was not a correctly spelled word, even though when pronounced it sounds like a real word. Example of a yes item is "bus." Example of a no item, a homonym to the real word "ear", is the foil "eer."

Task 3 syntactic judgments with and without homonym foils. The participant was instructed to press yes if the sentence could be a real sentence that is meaningful because all the words are spelled correctly and make sense in the sentence, but to press no if the sentence was not meaningful because all the words do not make sense in the sentence. Each sentence was presented for 3 seconds. The "no" items differed from the "yes" items by only one word which was a homonym foil. This is an example of a yes sentence: "The bee, which buzzes, can sting you." This is an example of a no sentence: "The bee, witch buzzes, can sting you."

Task 4 lexical judgments about true or fake affixes. The participant was instructed to press yes if the word has a true affix, but to press no if the word has the same spelling as an affix but was not an affix. This is an example of a yes item: "untie". This is an example of a no item: "under".

Task 5 syntactic judgments with and without affix foils. The participant was instructed to press yes if the bolded word could fit the sentence and the sentence was meaningful. This is an example of a yes item: "He was unfit physically." This is an example of a no item: "He was unfitted physically."

Task 6 multi-sentence text judgments. The participant was instructed to read each of the four sentences that will appear on the monitor one at a time and then press yes if the fifth sentence is true based on the four prior sentences read, or no if it is false. Five written sentences were presented on the monitor one at a time (each presented for a constant time interval). The last sentence was always a statement about the text accumulated so far that can be answered true (yes) or false (no).

Example set for a "true" response follows:

Sentence 1: John handed Bill a note.

Sentence 2: It was from Sarah.

Sentence 3: Sarah had written that she wanted to talk to Bill.

Sentence 4: Bill frowned when he read the note.

Sentence 5: True or False? (press key to answer)

Bill was not pleased with what Sarah had written. (True)

Example set for a "false" response follows:

Sentence 1: Tomorrow is the day of the picnic.

Sentence 2: If it rains, the picnic will be cancelled.
Sentence 3: Amy listens to the weather report.

Sentence 4: She hopes it will rain.

Sentence 5: True or False? (press key to answer)

Amy wants to go to the picnic. (False)

\section{fMRI writing tasks}

During the fMRI writing tasks, a mirror system enabled the participant in the scanner to see the instructions and task on a screen. It was possible to study writing during imaging by using an MRIcompatible stylus, which allows participants to write while in the scanner and stores what they write concurrently and is registered in time with the fMRI data acquisition for subsequent analyses [31]. Two kinds of transcription tasks were included-one involving only sublexical handwriting and one involving lexical spelling. One kind of translation task was included (planning). A resting condition was analyzed in some of the prior fMRI connectivity studies [32] before the clustering coefficient graph analyses; this condition is thought to involve self-guided thinking during mind wandering [33]. However, it was not included in the current study because it does not involve an experimenter designed task nor is it hypothesized to be related to adaptive control or the working memory components in the language learning mechanism. The tasks and writing pad recordings were all programmed, timed, and coordinated with the scanner triggers using E-prime and in-house LabView software. During the fMRI writing tasks the same parameters were used as with the resting condition for fMRI reading tasks but with 387 dynamic scans, total acquisition time 13:08 $\mathrm{min} / \mathrm{s}$.

$f M R I$ alphabet writing task. The participant was instructed to write the letter that follows a visually displayed letter in alphabet order. There were 6 seconds of instruction for the alphabet task. The alphabet writing task lasted for 4 minutes and was self-paced. After the visual display of the first letter, the participant wrote the next letter in the alphabet. When the participant lifted the pen off the tablet, the next visual display appeared, and the process repeated until the 4 minutes were completed.

fMRI spelling fill in letter task. The participant was instructed to fill in the blank in a visually displayed letter string to create a correctly spelled word. There were 6 seconds of instruction for spelling, followed by the spelling task that lasted for $4 \mathrm{~min}$ and was self-paced. After the first visual display, the participant wrote a letter in the blank to complete the word spelling. When the participant lifted the pen off the tablet, the second visual display appeared, and the process repeated until the 4 minutes were completed.

fMRI planning task. Just before exiting the scanner, the participant was instructed through ear phones to begin planning a composition to be written by pen and paper outside the scanner. The topic was advice to astronauts about writing while traveling in outer space. After scanning during planning was completed, the participant exited the scanner and wrote the composition on that topic with pen and paper.

\section{Functional connectivity analyses}

Functional images were corrected for motion using FSL MCFLIRT [34], and then high-pass filtered at sigma $=20.83$ (in units of fMRI volumes, not seconds). Average motion score (mean absolute displacement) was computed for each participant. Spikes were identified and removed using the default parameters in AFNI's 3dDespike. Slice-timing correction was applied with FSL's slicetimer and spatial smoothing was performed using a 3D Gaussian kernel with FWHM = $4.0 \mathrm{~mm}$. Time series motion parameters and the mean signal for eroded 
Richards TL (2017) Self-government of complex reading and writing brains informed by cingulo-opercular network for adaptive control and working memory components for language learning

( $1 \mathrm{~mm}$ in $3 \mathrm{D})$ masks of the lateral ventricles and white matter (derived from running FreeSurfer's recon-all on the T1-weighted image) were analyzed. For each participant average motion score (mean absolute displacement) was used to decide whether to keep the fMRI data or throw it out (greater than one voxel size $3.0 \mathrm{~mm}$ was the threshold to exclude). Data were also excluded if the standard motion regressors were unable to take out the effects of motion.

Co-registration of functional images to the $\mathrm{T} 1$ image was performed using boundary-based registration based on a white matter segmentation of the T1 image through epi_reg in FSL. The MPRAGE structural scan was segmented using FreeSurfer software; white matter regressors were used to remove unwanted physiological components. Software was written in gfortran to compute a $68 \times 68$ correlation matrix which was used in the Brain Connectivity Toolbox. This $68 \times 68$ correlation matrix was made by finding the cross correlation between the fMRI time series signal between brain regions where each of the 68 brain regions was found using the cortical regions of the MORI atlas [35]. This atlas contains 176 brain regions as shown in http://www. spl.harvard.edu/publications/item/view/1883. A provided file called JHU_MNI_SS_WMPM_Type-I_SlicerLUT.txt contains 176 brain region names, but only the 68 cortical regions of the brain related to the specific aims of the current study were used for the analyses. See Appendix.

The individual space of the fMRI scan was co-registered to this atlas using FSL FLIRT software. Then to investigate the nodes of the CO network, 16 regions of significant fMRI connectivity bilaterally were identified all of which were previously shown to be significant for multiple comparison corrections as processed using FSL software randomise: left and right cingulate gyrus, left and right superior frontal gyrus, left and right middle frontal gyrus, left and right inferior frontal gyrus, left and right superior temporal gyrus, left and right insula, left and right cingulum (near cingulate gyrus), and left and right cingulum (near hippocampus). Matlab software called "Brain Connectivity Toolbox", https:// sites.google.com /site/bctnet/construction, was used to perform the complex network analysis/graph theory analysis, as described in Rubinov and Sporns (2010) [7]. The clustering coefficients of the regions within the $\mathrm{CO}$ network were based on the full-brain connectivity network regions thresholded at $10 \%$ sparsity. Although there are multiple approaches to thresholding a network, a value of $10 \%$ sparsity threshold results in valuable network testing [36] and was employed in the current study.

\section{Behavioral measures of adaptability and working memory}

BASC 2 Adaptive Control. Parents completed The Behavior Assessment System for Children, Parent Rating Scale (BASC2-PRS) [12] which yields $T$-scores for the Adaptive Skills Composite. Because there were more boys than girls in the sample, only the $T$-scores based on norms for the separate genders were used for the purpose of correlating graph clustering coefficients with parent ratings of Adaptive Skills.

Phonology coding. For the Comprehensive Test of Phonological Processing (CTOPP) [37] Nonword Repetition subtest (test-retest reliability 0.70 ), the task is to listen to an audio recording of nonwords, which are pronounced one at a time, and then repeat exactly the heard oral nonword, which contains English sounds but has no meaning; the score is a measure of phonology coding (word form storage and processing). The raw score for accuracy is converted into a scaled score for age $(M=10, S D=3)$. Research has shown that phonological coding has both a genetic basis [38] and a brain basis [39].

Orthotactics for letter sequencing and orthography coding for words. For the Test of Orthographic Competence TOC Word Scrambles (test-retest reliability 0.88 to 0.90 ), the task is to rearrange letters in a scrambled word to create a correctly spelled real word (word-specific spelling) [40]. Raw scores are converted to scaled scores for age ( $M=10$, $S D=3$ ) on this orthographic coding measure sensitive to orthotactics for letter identity, position, and sequencing [41]. For the Test of Silent Word Reading Fluency (TOSWRF) [42], (test-retest reliability 0.92) the task is to mark the word boundaries in a series of letters arranged in rows. The score is the number of correctly detected and marked word boundaries in 3 minutes, which is a measure of orthography coding (word form storage and processing); raw scores are converted to standard scores for age $(M=100, S D=15)$. Orthographic spelling has also been shown to have both a genetic basis [43] and a brain basis [32,39].

Morphology coding. For the experimenter-designed Comes From $z$-score for grade, which is a measure of morphology coding (word form storage and processing), the task is to judge whether or not a heard and viewed word is derived from a base word [44]. Example items follow: "Does corner come from corn?" "Does builder come from build?" In both cases the words in a pair contain a common spelling ("er" ending), but it may or may not function as a morpheme that transforms a base word. Raw scores are transformed to $z$-scores $(M=0$, $S D=1$ ) based on research norms for elementary and middle school grades. Thus, this measure, which has been shown to have a brain basis [39], is a behavioral analogue of the fMRI affixed word reading task 4 (judgments about affixes).

Syntax coding. The Clinical Evaluation of Language Function $4^{\text {th }}$ Edition CELF IV [45] Formulated Sentence subtest (test-retest reliability 0.62 to 0.71 ) requires constructing an oral sentence from three provided words for each item; it yields scaled scores for age $(M=10$ and $S D=3)$.

Phonological loop. For Rapid Automatic Letter Naming RAN (testretest reliability .90) [46], which is a measure of phonological loop for cross-code integration in language learning [47] and has a genetic basis [48], the task is to name lower case printed letters arranged in rows. The total score is the time required to name all the letters in all the rows. It is converted to a standard score for age $(M=100, S D=15)$.

Orthographic loop. On the Alphabet Writing Task, an experimenterdesigned test, children are asked to handwrite in manuscript (unjoined letters) the lower case letters of the alphabet from memory as quickly as possible in alphabetic order, but to make sure others can identify the letters [3]. The raw score is the number of letters that are legible and in correct order during the first 15 seconds. The raw score is converted to a $z$-score $(M=0, S D=1)$, based on research norms for grade (inter-rater reliability 0.97). This task has been shown to have a brain basis [32].

Focused attention. For Delis Kaplan Executive Functions D-KEFS [49] Color Word Form Inhibition (reliability ranges from 0.62 to 0.76 ), based on the classic Stroop task, the task is to read orally words that name a color and are printed in black ink, and then to name the color of the ink in which words that name a color are printed but the ink color conflicts with the color the word names (e.g., the word "red" written in green ink). The difference in time for reading the words in black and naming the conflicting-ink color word is an index of focused attention (inhibition of irrelevant information and focus on relevant information). Raw scores are converted to scaled scores for age $(M=10, S D=3)$.

Switching Attention. For Rapid Automatic Switching (RAS)-letters and numerals (test-retest reliability 0.90) [46], the task is to name alternating lower case printed letters and written numerals arranged in rows. The total score is the time required to name all the alternating letters and numerals in all the rows and provides a measure of switching attention. RAS has also been shown to have a genetic basis [48]. The raw score is converted to a standard score $(M=100$ and $S D=15)$. 
Richards TL (2017) Self-government of complex reading and writing brains informed by cingulo-opercular network for adaptive control and working memory components for language learning

Theory and evidence-driven correlations. All correlation analyses in Tables 1 and 2 were theory driven and informed by empirical findings in prior research about both the $\mathrm{CO}$ network and working memory phenotypes in children with and without specific learning disabilities in written language (SLDs-WL). They were not exploratory [50]. However, only correlations significant at $p$ in the 0.009 to 0.000 range are reported in Tables 1 and 2 to inform future research on the replicability of the current findings. Although conventional approaches might report these as $p<.01$ or $p<.001$ the actual $p$-values give a more precise sense of the range in probabilities.

\section{Results}

Although the clustering coefficients informed by graph theory were conducted only for the 16 brain regions in the CO network listed above, the results of these analyses identified graph clustering coefficients in the $\mathrm{CO}$ network having connectivity with many of the cortical regions in the 68 brain regions identified in the initial connectome analyses.

\section{Research aim 1 and hypothesis: Brain-behavior relationships in adaptive control}

As shown in Table 1, at time 1 the BASC 2 Parent Ratings of Adaptivity were correlated with the clustering coefficients for $\mathrm{CO}$ network and its connections with other regions in the connectome on three fMRI reading tasks: Phoneme-Grapheme Correspondences in Right Middle Frontal Gyrus, True Affixes versus Foils in Left Cingulum (near Hippocampus), and Reading Comprehension for Sentences with and without Homonym Foils in Right Cingulate Gyrus. As shown in Table 2, at time 1 the BASC 2 Parent Ratings of Adaptivity were also correlated with the fMRI Alphabet Writing Task in Left Cingulate Gyrus.

Summary of findings related to first research aim. Generally the results supported the first tested hypothesis that cingulum-operculum (CO) network is involved in adaptive control for written language learning. The behavioral parent ratings of BASC 2 Adaptivity were related to clustering coefficients of the $\mathrm{CO}$ network involved in adaptive control on three of the six fMRI reading tasks spanning the subword, word, and sentence levels of reading. In addition, these parent ratings of adaptivity were also related to clustering coefficients of the CO network on one fMRI writing task involving accessing, finding, retrieving, and producing ordered letters in the alphabet from memory. However, the clustering coefficients in the CO network were not shown to be involved in all fMRI reading and fMRI writing tasks included in the current study. Thus, the adaptive control function of the $\mathrm{CO}$ network may be related to the nature of the reading or writing task. It is also important to note that the $\mathrm{CO}$ clustering coefficients showed connectivity with other regions in the connectome, pointing to the probable role of this adaptive control network in the brain's government of other regions in the connectome.

Table 1. Significant Correlations between Graph Cluster Coefficients for Statistically Significant fMRI Connectivity and Working Memory Components in the Language Learning System for fMRI Reading Tasks at Different Levels of Language

\begin{tabular}{|c|c|c|c|c|c|c|c|c|}
\hline FMRI Task & $\begin{array}{c}\text { Time1 Brain } \\
\text { Region }\end{array}$ & $\begin{array}{c}\text { Correlated With } \\
\text { Behavior }\end{array}$ & $\mathbf{r}$ & $\mathbf{p}$ & Time2 Region & $\begin{array}{c}\text { Correlated } \\
\text { With Behavior }\end{array}$ & $\mathbf{r}$ & $\mathbf{p}$ \\
\hline \multirow[t]{4}{*}{ Phoneme } & Right MFG & BASC2 Adaptivity & 0.55 & 0.000 & & & & \\
\hline & Right CingulumH & Phonology Coding & 0.43 & 0.005 & & & & \\
\hline & & Orthography Coding & 0.43 & 0.007 & & & & \\
\hline & & Letter Sequencing & 0.46 & 0.007 & & & & \\
\hline \multirow[t]{2}{*}{ Spelling } & Left STG & Focused Attenton & -0.42 & 0.007 & Left CingulumH & Phonological Loop & -0.43 & 0.005 \\
\hline & Left CingulumC & Letter Sequencing & 0.49 & 0.009 & & & & \\
\hline \multirow[t]{4}{*}{$\begin{array}{c}\text { Sentence } \pm \\
\text { Homonym Foils }\end{array}$} & Left SFG & Phonological Loop & 0.43 & 0.006 & Left STG & Orthographic Loop & 0.63 & 0.000 \\
\hline & & Switching Attention & 0.48 & 0.002 & Left CingulumC & Orthographic Loop & 0.71 & 0.000 \\
\hline & Left CingulumC & Morphology Coding & -0.58 & 0.000 & Right STG & Orthographic Loop & 0.58 & 0.000 \\
\hline & Right CingulumC & Letter Sequencing & 0.58 & 0.000 & & & & \\
\hline \multirow[t]{5}{*}{ True Affix } & Left STG & Orthography Coding & 0.51 & 0.001 & Left Cingulate & Letter Sequencing & 0.47 & 0.007 \\
\hline & Left CingulumC & Focused Attention & -0.43 & 0.005 & Left STG & Phonology Coding & 0.43 & 0.006 \\
\hline & Left CingulumH & BASC2 Adaptivity & 0.50 & 0.001 & Right Cingulate & Letter Sequencing & 0.48 & 0.006 \\
\hline & Right STG & Orthography Coding & 0.44 & 0.005 & Right CingulumC & Orthographic Loop & 0.83 & 0.000 \\
\hline & Right Insula & Letter Sequencing & 0.50 & 0.003 & & & & \\
\hline \multirow[t]{2}{*}{ Sentence \pm Affix Foil } & Left CingulumC & Syntax Coding & -0.41 & 0.006 & Right IFG & Phonological Loop & -0.69 & 0.000 \\
\hline & Right CingulumH & Letter Sequencing & 0.47 & 0.006 & & & & \\
\hline \multirow[t]{8}{*}{ Multi-Sentence } & Left MFG & Orthography Coding & -0.42 & 0.008 & Left SFG & Phonological Loop & 0.69 & 0.000 \\
\hline & Left Insula & Phonology Coding & 0.42 & 0.006 & & & & \\
\hline & & Orthography Coding & 0.45 & 0.004 & & & & \\
\hline & & Letter Sequencing & 0.46 & 0.007 & & & & \\
\hline & Right MFG & Morphology Coding & -0.59 & 0.000 & & & & \\
\hline & Right STG & Ortholgraphy Coding & -0.51 & 0.001 & & & & \\
\hline & Right Insula & Letter Sequencing & 0.45 & 0.008 & & & & \\
\hline & Right CingulumH & Letter Sequencing & 0.46 & 0.007 & & & & \\
\hline
\end{tabular}

Notes:

Phoneme $=$ Phoneme Grapheme; Spelling $=$ Correct spelling or Homonym foil; MFG $=$ middle frontal gyrus; STG $=$ superior temporal gyrus; CingulumH $=$ Cingulum part near the hippocampus; CingulumC $=$ Cingulum part near the cingulate gyrus.

Notes. Only correlations significant in the $\mathrm{p} \leq .001$ or in the .002 through .009 range are reported in the table. See text for description of these measures: phonology coding (CTOPP Nonword Repetition), orthography coding (TOSWRF), letter sequencing (TOC anagrams), morphology coding (Comes From), sentence syntax (syntactic coding) phonological loop (RAN), orthographic loop (RAS), Focused Attention (D-KEFS Inhibition), and switching attention (BASC2 Adaptivity). All nine components of multi-component working memory supporting language learning were significantly correlated with at least one cluster coefficient for the cingulo-operculum network at $p \leq .001$ or in the $p=.002$ through .009 range. 
Richards TL (2017) Self-government of complex reading and writing brains informed by cingulo-opercular network for adaptive control and working memory components for language learning

Table 2. Significant Correlations between Graph Cluster Coefficients for Statistically Significant fMRI Connectivity and Working Memory Components in the Language Learning System for fMRI Transcription and Translation Tasks See Table Notes.

\begin{tabular}{|c|c|c|c|c|c|c|c|c|}
\hline FMRI Task & $\begin{array}{l}\text { Time1 Brain } \\
\text { Region }\end{array}$ & $\begin{array}{l}\text { Correlated With } \\
\text { Behavior }\end{array}$ & $\mathrm{r}$ & $\mathrm{p}$ & Time2 Region & $\begin{array}{l}\text { Correlated } \\
\text { With } \\
\text { Behavior }\end{array}$ & $\mathrm{r}$ & $\mathrm{p}$ \\
\hline \multirow[t]{5}{*}{ Alphabet } & Left Cingulate & BASC2 Adaptivity & 0.53 & 0.001 & Right Cingulate & Phonology Coding & 0.43 & 0.008 \\
\hline & Left SFG & BASC2 Adaptivity & -0.42 & 0.009 & Right Cingulate & Orthography Coding & 0.35 & 0.004 \\
\hline & Left SFG & Morphology Coding & 0.43 & 0.008 & Right MFG & Switching Attention & -0.44 & 0.006 \\
\hline & Right Cingulate & Orthography Coding & 0.45 & 0.006 & Right CingulumH & Focused Attention & -0.52 & 0.001 \\
\hline & Right SFG & Orthographic Loop & 0.48 & 0.002 & & & & \\
\hline \multirow[t]{4}{*}{$\begin{array}{l}\text { Spelling Fill in the } \\
\text { Blank }\end{array}$} & Left SFG & Orthography Coding & 0.46 & 0.005 & Left MFG & Morphology Coding & 0.46 & 0.005 \\
\hline & Right SFG & Orthographic Loop & -0.45 & 0.004 & Right CingulumC & Orthographic Loop & 0.66 & 0.000 \\
\hline & Right IFG & Syntactic Coding & 0.42 & 0.009 & Left SFG & Morphology Coding & 0.46 & 0.005 \\
\hline & & & & & Right CingulumC & Orthographic Loop & 0.66 & 0.000 \\
\hline \multirow[t]{2}{*}{ Planning } & Right Cingulate & Morphology Coding & -0.43 & 0.007 & Right Insula & Orthographic Loop & 0.66 & 0.000 \\
\hline & & & & & Right CingulumC & Orthographic Loop & 0.73 & 0.000 \\
\hline
\end{tabular}

Notes. Alphabet=Writing the next letter in the alphabet. See Text for description of fMRI tasks and clinical measures and Table 1 notes which also apply to results reported in Table 2.

\section{Research aim 2-first hypothesis: Time 1 behavior-brain relationships for working memory in language learning and CO clustering coefficients for adaptive control}

As shown in Tables 1 and 2, at Time 1 all the working memory components (behavioral phenotypes) were correlated with a clustering coefficient for $\mathrm{CO}$ network on at least one fMRI reading or fMRI writing task. In contrast to the results in Table 1 organized by fMRI reading tasks at time 1 and time 2 and in Table 2 organized by fMRI writing tasks at time 1 and time 2 , the results in this section are summarized by working memory components and fMRI tasks assessed at time 1 .

Phonological coding. This working memory behavioral phenotype was correlated with clustering coefficients on the following fMRI reading tasks: the fMRI phoneme-orthographic task in right cingulum (near hippocampus) and the fMRI text reading comprehension task in left insula, but there were no significant correlations on fMRI writing tasks.

Orthography coding for words. This working memory behavioral phenotype based on written words detected in letter series was correlated with clustering coefficients on the following fMRI reading and writing tasks: the fMRI phoneme-orthographic task in right cingulum (near hippocampus), fMRI affixes task in left and right superior temporal gyrus, fMRI text reading comprehension task in left middle frontal gyrus, left insula and right superior temporal gyrus, and fMRI alphabet writing task in right cingulate gyrus, fMRI alphabet task and right cingulate, and fMRI spelling-fill in the blank in left and right superior frontal gyrus.

Orthotactic coding for letter identity, position, and sequencing in English words. This working memory behavioral phenotype based on anagrams (changing letter sequence to create correctly spelled word for letter identity, position, and sequencing in written English) was correlated with clustering coefficients on the following fMRI reading tasks: fMRI word reading (word-specific spelling judgment task) in left cingulum (near cingulate gyrus), fMRI reading comprehension for sentences with and without homonym foils in right cingulate gyrus, fMRI affix judgment task in right insula, fMRI reading comprehension for sentences with and without affix foils in right cingulum (near hippocampus), and fMRI text comprehension in left insula, right insula, and right cingulum (near hippocampus). However, there were no significant correlations on fMRI writing tasks.

Morphological coding. This working memory behavioral phenotype was correlated with clustering coefficients on these fMRI reading taskssentence reading comprehension with and without homonym foils in left cingulum (near lingual gyrus), and text reading comprehension in right middle frontal gyrus, and these fMRI writing tasks-alphabet writing in left superior frontal gyrus, and fMRI planning in right cingulate gyrus.

Syntax coding. This working memory behavioral phenotype was correlated with clustering coefficients on this fMRI reading tasksentence reading comprehension with and without affix foils in left cingulum (near cingulate gyrus), and this fMRI writing taskspelling-fill in the blank in right inferior frontal gyrus.

Phonological loop. This working memory behavioral phenotype was correlated with clustering coefficients on the following fMRI reading task: sentence reading comprehension with and without homonym foils in left superior frontal gyrus. There were no significant correlations on fMRI writing tasks.

Orthographic loop. This working memory behavioral phenotype was correlated with clustering coefficients on the following fMRI writing tasks: alphabet writing with right superior frontal gyrus, and spelling-fill in the blank in right superior frontal gyrus. There were no significant correlations on fMRI reading tasks.

Focused attention. This working memory behavioral phenotype was correlated with clustering coefficients on the following fMRI reading tasks: word reading (word-specific spelling) in left superior temporal gyrus and word reading (true affix) in left cingulum (near cingulate gyrus). There were no significant correlations on fMRI writing tasks.

Switching attention. This working memory behavioral phenotype was correlated with clustering coefficients on the following fMRI reading task: sentence reading comprehension with and without homonym foils in left superior frontal gyrus. There were no significant correlations on fMRI writing tasks.

Summary of results for correlations between working memory behavioral phenotypes andf cluster coefficients of the CO network at time 1. Collectively, the results showed significant correlations for all working memory components, shown in prior assessment, genetic, and/or brain research to be related to reading and writing acquisition in the grade range investigated in the current study, with at least one fMRI clustering coefficient in the CO network. However, which working memory components were correlated with which clustering coefficients depended on whether fMRI reading or fMRI writing tasks were analyzed, or which specific fMRI reading or fMRI writing task was analyzed. Thus, there was general support for the hypothesis that working memory components at the behavioral level of analysis are correlated with clustering coefficients in the $\mathrm{CO}$ network at the brain 
Richards TL (2017) Self-government of complex reading and writing brains informed by cingulo-opercular network for adaptive control and working memory components for language learning

level of analysis, but relationships need to be qualified by specific working memory components and specific fMRI reading and writing tasks. Nevertheless, the results support a model in which both the $\mathrm{CO}$ adaptive control network and working memory phenotypes support the brain's government of the complex connectome involved in written language learning and use (reading and writing).

\section{Research aim 2-second hypothesis: Time 1 to time 2 changes in correlations between behavioral working memory Phenotypes and $\mathrm{fMRI}$ clustering coefficient}

As shown in Tables 1 and 2, at Time 2 the pattern of correlations between working memory measures at the behavioral level and $\mathrm{CO}$ network clustering coefficients at the brain level changed compared to Time 1 on the various fMRI reading or writing tasks. It is important to keep in mind that none of these correlations refer to a region of interest for BOLD activation but rather to a region with a significant clustering coefficient identified on basis of $\mathrm{CO}$ network, which may also have connections with other cortical regions in the connectome. Findings for this research aim are organized by fMRI reading task and fMRI writing task.

fMRI phoneme-grapheme correspondence judgments. In contrast to time 1 when both the phonological coding and orthographic coding phenotypes were correlated with this fMRI task, no correlations between phonological or orthographic coding and the clustering coefficients were significant at time 2 . One possible interpretation of this finding is that the instruction emphasizing grapheme-phoneme correspondences in the reading direction and phoneme-grapheme correspondences in the spelling direction greatly reduced or eliminated the need to focus limited working memory resources on these correspondences.

fMRI word reading (word-specific spelling) judgments (correctly spelled versus homonym foil). In contrast to time 1 when focused attention and orthotactic coding (letter sequencing) were correlated with this fMRI reading task, at time 2 only phonological loop was correlated with this word reading task. One possible interpretation of this finding is that the intervention, which included learning activities that facilitated paying attention to orthotactic coding, also facilitated response to the learning activities on grapheme-phoneme correspondences. Thus, they were better able to differentiate correct word-specific spellings and homonym foils.

fMRI word reading affix judgments (true affixes versus affix foils). The pattern of correlations changed following instruction in these ways. First, some correlations involved a change in which behavioral phenotype was correlated with the same brain region: in left superior temporal gyrus from orthography word coding to phonology word coding, and in the left cingulum (near cingulate gyrus) from focused attention to orthotactic coding (letter sequencing). Second, one change involved which brain regions a given working memory behavioral phenotype was correlated with: orthotactic coding (letter sequencing) in right insula at time 1 but right and left cingulate gyrus at time 2 . Third, one change involved which brain region previously correlated with a given behavioral phenotype at time 1 was no longer correlated at time 2: word orthography coding in right superior temporal gyrus at time 1 but no brain region at time 2. Fourth, a new correlation not observed at time 1 emerged at time 2: in right cingulum (near cingulate gyrus) with the orthographic loop behavioral phenotype. One possible interpretation of these collective findings is that instruction on how morphology interrelates with orthography and phonology for word spelling had changed the mental government of the brain during affix judgments, which may still be evolving for these students.
fMRI reading comprehension judgments for sentences with and without homonym foils. Correlations at time 1 for phonological loop and switching attention behavioral phenotypes with left superior frontal gyrus, morphology behavioral phenotype with left cingulum (near lingual gyrus), and orthotactic coding (letter sequencing) behavioral phenotype with right cingulum (near cingulate gyrus) were not observed at time 2. Rather correlations with a new behavioral phenotype emerged-orthographic loop-with left superior frontal gyrus, left cingulum (near cingulate gyrus), and right superior temporal gyrus. One possible interpretation of these findings is that the specialized instruction emphasizing instruction in letter formation and writing about reading source materials supported development of the orthographic loop.

fMRI reading comprehension judgments for sentences with and without affix foils. Correlations at time 1 for the sentence coding behavioral phenotype with left cingulum (near cingulate gyrus) and orthotactic coding (letter sequencing) behavioral phenotyping with right cingulum (near hippocampus) were not observed at time 2 . Rather, a new correlation emerged for the phonological loop behavioral phenotype with right inferior frontal gyrus. One possible interpretation of this finding is that following the specialized instruction emphasizing both morphology and sentence syntax, participating students engaged in covert oral reading of sentences to evaluate the plausibility of the various words, one of which may have had an affix foil for the syntactic context.

fMRI text reading comprehension judgments. None of the correlations at time 1 on this task were observed at time 2: orthographic word coding with left middle frontal gyrus, left insula, or right temporal gyrus; orthotactic coding (letter sequencing) with left and right insula or right cingulum (near hippocampus); phonology coding with left insula; or morphological coding with right middle frontal gyrus. A correlation with phonological loop emerged at time 2 with left superior frontal gyrus. A possible interpretation is that correlations emerged between the phonological loop with cluster coefficients in a brain region involved in translation of thought into language (superior frontal gyrus). Not only overt but also covert phonological loop of working memory may support holding multiple sentences in working memory until the full comprehension process is completed for understanding stated information and making necessary inferences.

fMRI alphabet writing. Correlations at time 1 not observed at time 2 included morphological coding with left superior frontal gyrus, and orthographic loop with right superior frontal gyrus. At time 2, orthography coding was still correlated with right cingulate gyrus but correlations emerged between phonology coding with right cingulate gyrus; focused attention with right cingulum (near hippocampus); and switching attention with right middle frontal gyrus. A possible interpretation is that the instruction, which emphasized naming the letters for retrieving them from memory, and focusing attention on each stroke in letter formation and switching attention between sequential strokes, resulted in the reorganization of the mental government for writing ordered letters from memory.

fMRI spelling-fill in the blank. Correlations at time 1 not observed at time 2 included orthography word coding with left superior frontal gyrus and syntactic coding with right inferior frontal gyrus. The correlation for orthographic loop found at time 1 with right superior frontal gyrus was found with right cingulum (near cingulate gyrus) at time 2 . New correlations emerged for the first time at time 2 for morphology word coding with left superior frontal gyrus and left middle frontal gyrus. A possible interpretation of these findings is that the specialized instruction in handwriting and word spelling contributed to changes in the brain's government for letter writing 
Richards TL (2017) Self-government of complex reading and writing brains informed by cingulo-opercular network for adaptive control and working memory components for language learning

to fill in the blank to create a correctly spelled word; emergence of the correlations with morphology word coding may show its role in integrating single letters and lexical units in word spelling.

fMRI planning. The correlation for morphology word coding observed at time 1 with right cingulate gyrus was not observed at time 2. However, correlations emerged at time 2 for orthographic loop with right insula and with right cingulum (near cingulate gyrus). A possible interpretation of these findings is that before instruction, writers were focused during planning for their compositions on the translation of their thoughts into word meaning, but after instruction aimed at both transcription and translation, the participants focused more on integrating their transcription processes (orthographic loop) with planning for translation, before engaging in written composing.

Summary of findings for changing patterns of correlations between time 1 and time 2. Support was found for the second hypothesis related to changes in the pattern of these relationships. However, this conclusion has to be qualified by the nature of the behavioral phenotype and the nature of the fMRI reading or fMRI writing task. Nevertheless, these changes in the relationships between the working memory phenotypes and specific clustering coefficients in the CO network from before to after instruction support a model in which brain RTI following instruction involves not only changes in specific regions or networks involved in specific language functions, but also in the brain government for self-regulating language learning and use. This dynamic brain government, which can be modified in response to instruction, appears to draw on both the CO adaptive control network and the working memory components supporting language learning. As a summary, Figure 1 shows brain regions where there were significant correlations with scores at time 2 but not at time 1 for the reading tasks. Figure 2 shows brain regions where there were significant correlations with scores at time 2 but not at time 1 for the writing tasks.
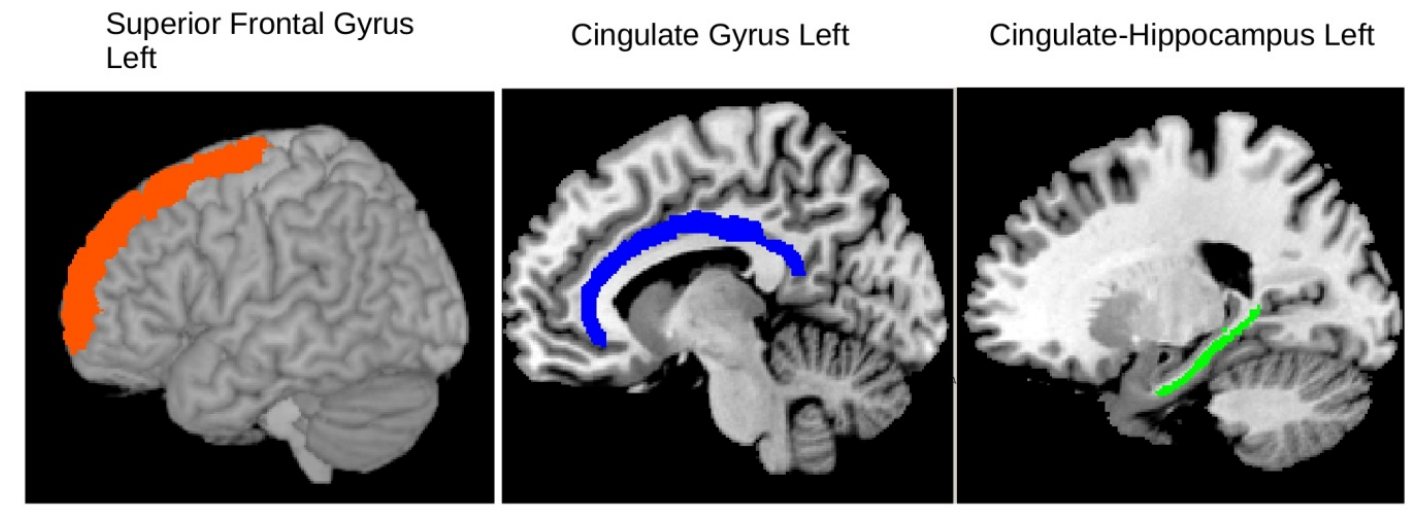

Figure 1 - fMRI reading task

Brain regions, organized in order of top-middle-lower brain locations, for which there were significant correlations with an fMRI cluster coefficient and working memory component at time 2 but not at time 1 .

Left superior frontal gyrus shown in red for which there was a significant correlation between fMRI cluster coefficient (reading multi-sentence task) and phonological loop scores at time 2 . Left cingulate gyrus shown in blue for which there was a significant correlation between fMRI cluster coefficient (sentence reading with and without homonym foils task) and orthographic loop scores at time 2 .

Left cingulum- hippocampus shown in green for which there was a significant correlation between fMRI cluster coefficient (word reading task-correct spellings or homonym foils) and phonological loop scores at time 2 .

Middle Frontal Gyrus Left

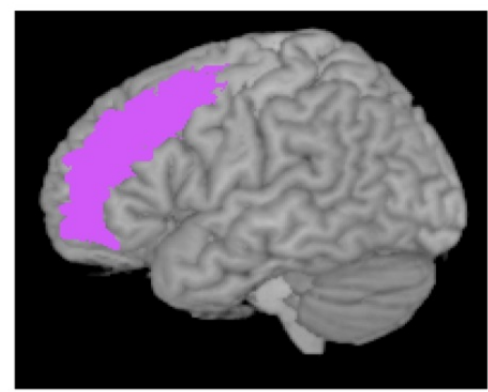

Cingulate Gyrus Right

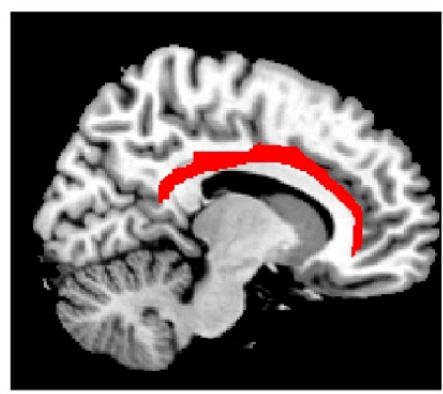

Figure 2 - fMRI writing tasks

\section{Cingulate-Hippocampus Right}

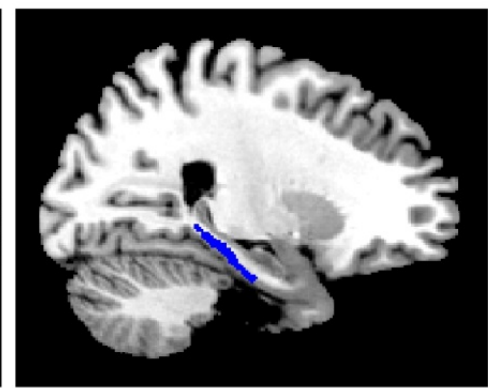

Brain regions, organized in order of top-middle-lower brain locations, for which there were significant correlations with an fMRI cluster coefficient and working memory component at time 2 but not at time 1 .

Left middle frontal gyrus shown in purple for which there was a significant correlation between fMRI cluster coefficient (spelling - fill letter in blank task) and morphological coding scores at time 2 .

Right cingulate gyrus shown in red for which there was a significant correlation between fMRI cluster coefficient (writing planning task) and orthographic loop scores at time 2.

Right cingulum-hippocampus shown in blue for which there was a significant correlation between fMRI cluster coefficient (writing next letter in alphabet task) and focused attention scores at time 2 . 
Richards TL (2017) Self-government of complex reading and writing brains informed by cingulo-opercular network for adaptive control and working memory components for language learning

\section{Discussion and conclusions}

On the one hand, the findings of the current study illustrate the complexity of the human connectome [7] for both the reading and writing brain during middle childhood and early adolescence. On the other hand, they illustrate the complexity of the mental government for managing the complex multi-leveled reading and multi-task writing systems, especially in an era when brain imaging methods allow more precise measurement of localization within functional networks. Accordingly, the findings not surprisingly support the longstanding programmatic research of Posner and colleagues, showing that a single executive function alone cannot account for the orchestration of mind in the brain's reading system [51].

In addition, the results based on correlations between BASC 2 parent ratings for Adaptivity and correlations with clustering coefficients involving the cingulum-operculum (CO) network on three fMRI reading tasks (subword, word, and syntax levels) and one fMRI orthographic loop task (writing the next letter in ordered alphabet), extend prior research supporting the adaptive control function of the $\mathrm{CO}$ network for aural language [8] to language by eye (reading) and language by hand (writing). The results based on correlations between the behavioral measures of working memory and clustering coefficients of the CO network for various fMRI reading or writing tasks provide converging evidence that both the working memory components and adaptive control of the $\mathrm{CO}$ network support language learning during middle childhood and early adolescence. These correlations for working memory measures for phonological coding, orthographic coding, and morphological coding provide support for the orthography, phonology, and morphology in the inheritance lexicons for reading and spelling English, which is a morphophonemic orthography [52].

Yet, by examining these correlations both before and after instruction, which had resulted in improved reading and writing achievement [17] and reading brain RTI [15] and writing brain RTI [9], it was possible to show that the patterns of behavior-brain correlations related to the brain's government of language learning also change in response to specialized instruction. That instruction was aimed at all the levels of language in reading and transcription and translation in writing. Currently researchers often focus on explicit instruction in a single reading or writing skill rather than strategies for facilitating mental government of the complex reading and writing brains given limited working memory resources and adaptive control requirements necessary for managing the language learning process.

\section{Limitations and future research directions}

The sample size, while relatively small for some research topics, is not smaller than samples in many other brain imaging studies, especially those which have imaged participants before and after instructional intervention. Nevertheless, the findings provide evidence to encourage future studies with larger samples ascertained using comparable inclusion criteria. The current findings are limited, however, to the nature of the specialized instruction provided; additional research is needed on whether the nature of the instruction provided affects changes in brain's government of the complex reading and writing systems. Also, further research is needed on the possible interpretations of the specific kinds of changes in relationships between the working memory components and the brain's cluster coefficients following instruction.

Another limitation was not analyzing the genetic mediation for the brain's mental government of the complex reading and writing systems. Given the evidence that many of the working memory phenotype measures have been shown to have a genetic basis, not only specialized instruction but also genetic risk factors may mediate response to instruction and influence development of the brain's mental government. There is substantial evidence that genetic variants in the dopamine receptor $\mathrm{D} 4$ gene $(D R D 4)$ confer increased risk for ADHD [53-55]. However, the cognitive behavioral deficits in ADHD and reading disabilities are not the same [56]. In a sample selected for students with and without written language learning disabilities, the incidence of co-occurring ADHD was very low and normed measures of focused or supervisory attention or parent ratings of inattention were considerably better predictors of reading and writing outcomes than ADHD diagnosis [30]. Future research should address if DRD4 alleles for focused attention or supervisory attention or inattention ratings may be genetic mediators for self-regulating language learning. Different molecular markers of alleles associated with specific learning disabilities have been identified for each of the two hallmark phenotypes for each of these specific learning disabilities that affect language learning: dysgraphia, dyslexia, and OWL LD [57]. Thus, future research should also investigate whether the brain's mental government for language learning and use may be genetically mediated by alleles for both language and attention phenotypes.

Ultimately, increasing understanding of the genetic risk factors in the mental self-government of the complex reading and writing brains is relevant to developing personalized education, analogous to the important contribution of genetic analyses for personalized medicine [58]. Reading and writing brains must not only learn from teacherprovided explicit instruction but also learn to manage and govern their own self-regulated language learning. Both environmental and biological variables may influence how the reading and writing brains learn such mental self-government.

\section{Acknowledgements}

The current study was supported by grant P50HD071764 from the Eunice Kennedy Shriver National Institute of Child Health and Human Development (NICHD) at the National Institutes of Health (NIH) to the University of Washington Learning Disabilities Research Center. This interdisciplinary research has been a team effort. The first author headed the fMRI imaging data acquisition and analyses, the second author headed cross-center data analyses, the third and fourth contributed to the analyses, and/or interpretation of graph theory clustering coefficients, the fifth author headed the family genetics for the phenotypes and molecular genetics for the alleles, and the last author headed the reading and writing instructional intervention and collection of the behavioral data for working memory components in language learning mechanism and helped design the fMRI reading and writing tasks. The authors also thank William Nagy for assistance in developing the fMRI reading tasks and Mark Eckert and Jim Swanson for helpful consultation regarding this research.

\section{References}

1. Purcell JJ, Turkeltaub PE, Eden GF, Rapp B (2011) Examining the central and peripheral processes of written word production through meta-analysis. Front Psychol 2: 239. [Crossref]

2. van den Heuvel MP1, Sporns O (2011) Rich-club organization of the human connectome. J Neurosci 31: 15775-15786. [Crossref]

3. Berninger V (2009) Highlights of programmatic, interdisciplinary research on writing Learning Disabilities Research and Practice 24: 68-79. [Crossref]

4. Berninger V, Richards $T$ (2010) Inter-relationships among behavioral markers, genes, brain and treatment in dyslexia and dysgraphia. Future Neurol 5: 597-617. [Crossref]

5. Richards T, Nagy W, Abbott R, Berninger V (2016) Brain connectivity associated with cascading levels of language. J Syst Integr Neurosci 2. [Crossref] 
Richards TL (2017) Self-government of complex reading and writing brains informed by cingulo-opercular network for adaptive control and working memory components for language learning

6. Bullmore E, Sporns O (2009) Complex brain networks: graph theoretical analysis of structural and functional systems. Nat Rev Neurosci 10:186-198. [Crossref]

7. Rubinov M, Sporns O (2010) Complex network measures of brain connectivity: uses and interpretations. Neuroimage 52: 1059-1069. [Crossref]

8. Vaden KI Jr, Kuchinsky SE, Cute SL, Ahlstrom JB, Dubno JR, et al. (2013) The cingulo-opercular network provides word-recognition benefit. J Neurosci 33: 1897918986. [Crossref]

9. Richards TL, Berninger VW, Yagel K, Abbott R, Peterson D (2017) Brain's functional network clustering coefficient changes in response to instruction (RTI) in students with and without reading disabilities: Multi-leveled reading brain's RTI. Cogent Psychology Neuroscience (In Press).

10. Richards T, Berninger V, Fayol M (2012) The writing brain of normal child writers and children with writing disabilities: Generating ideas and transcribing them through the orthographic loop. A mosaic of new perspectives: New York: Psychology Press.

11. Wallis P, Richards T, Boord P, Abbott R, Berninger V (2017) Relationships between translation and transcription processes during fMRI connectivity scanning and coded translation and transcription in writing products after scanning in children with and without transcription disabilities. Creative Education, Special Issue on Teaching and Learning Language 8: 716-748. [Crossref]

12. Reynolds CR, Kamphaus RW (2004) BASC-2 Behavior Assessment System for Children (Second Edition) Circle Pines, MN: American Guidance Service.

13. Nielsen K, Haberman K, Richards T, Abbott R, Mickail T, et al. (2017) Emotiona and behavioral correlates of persisting specific learning disabilities in written language (SLDs-WL) during middle childhood and early adolescence. Journal of Psychoeducational Assessment (In Press).

14. Abel N, Berninger V (2017) Relationships between home literacy practices and schoo achievement: Implications for school-home collaboration and consultation practices. Journal of Educational and Psychological Consultation 1-26. [Crossref]

15. Richards TL, Berninger VW, Yagle KJ, Abbott RD, Peterson DJ (2017) Changes in DTI Diffusivity and fMRI Connectivity Cluster Coefficients for Students with and without Specific Learning Disabilities In Written Language: Brain's Response to Writing Instruction. J Nat Sci 3: e350. [Crossref]

16. Niedo J, Tanimoto S, Thompson RH, Abbott RD, Berninger VW (2016) Computerized Instruction in Translation Strategies for Students in Upper Elementary and Middle School Grades With Persisting Learning Disabilities in Written Language. Learn Disabil (Pittsbg) 21:14-30.

17. Tanimoto S, Thompson R1, Berninger VW2, Nagy W3, Abbott RD4 (2015) Computerized Writing and Reading Instruction for Students in Grades 4 to 9 With Specific Learning Disabilities Affecting Written Language. J Comput Assist Learn 31: 671-689. [Crossref]

18. Dosenbach NU, Visscher KM, Palmer ED, Miezin FM, Wenger KK, et al. (2006) A core system for the implementation of task sets. Neuron 50: 799-812. [Crossref]

19. Dosenbach NU, Fair DA, Miezin FM, Cohen AL, Wenger KK, et al. (2007) Distinct brain networks for adaptive and stable task control in humans. Proc Natl Acad Sci U S A 104: 11073-11078. [Crossref]

20. Rypma B, Berger JS, Prabhakaran V, Bly BM, Kimberg DY, et al. (2006) Neural correlates of cognitive efficiency. Neuroimage 33: 969-979. [Crossref]

21. Seeley WW, Menon V, Schatzberg AF, Keller J, Glover GH, et al. (2007) Dissociable intrinsic connectivity networks for salience processing and executive control. $J$ Neurosci 27: 2349-23456. [Crossref]

22. Song M, Zhou Y, Li J, Liu Y, Tian L, et al. (2008) Brain spontaneous functional connectivity and intelligence. Neuroimage 41:1168-1176. [Crossref]

23. Dosenbach NU, Fair DA, Cohen AL, Schlaggar BL, Petersen SE (2008) A dualnetworks architecture of top-down control. Trends Cogn Sci 12: 99-105. [Crossref]

24. Cocchi L, Zalesky A, Fornito A, Mattingley JB (2013) Dynamic cooperation and competition between brain systems during cognitive control. Trends Cogn Sci 17: 493501. [Crossref]

25. Chomsky N (1965) Aspects of the theory of syntax. Cambridge: MIT Press.

26. Berninger V, Swanson HL (2017) Role of working memory in the language learning mechanism by ear, mouth, eye, and hand in individuals with and without specific learning disabilities in written language. Working Memory and Neurodevelopmental Disorders: Abingdon, UK.

27. Venezky R (1970) The structure of English orthography. The Hague: Mouton. [Crossref]
28. Venezky R (1999) The American way of spelling. New York: Guilford. [Crossref]

29. Niedo J, Berninger VW (2016) Strategies typically developing writers use for translating thought into the next sentence and evolving text: Implications for assessment and instruction. Open Journal of Modern Linguistic 6: 276-292. [Crossref]

30. Berninger V, Abbott R, Cook C, Nagy W (2017) Relationships of attention and executive functions to oral language, reading, and writing skills and systems in middle childhood and early adolescence. Journal of Learning Disabilities 50: 434-449. [Crossref]

31. Reitz F, Richards T, Wu K, Boord P, Askren K, et al. (2013) A low-cost, computerinterfaced drawing pad for fMRI studies of dysgraphia and dyslexia. Sensors 13: 50995108. [Crossref]

32. Richards TL, Grabowski TJ, Boord P, Yagle K, Askren M, et al. (2015) Contrasting brain patterns of writing-related DTI parameters, fMRI connectivity, and DTIfMRI connectivity correlations in children with and without dysgraphia or dyslexia. Neuroimage Clin 8: 408-421. [Crossref]

33. Raichle ME, MacLeod AM, Snyder AZ, Powers WJ, Gusnard DA, et al. (2001) A default mode of brain function. Proc Natl Acad Sci U S A 98: 676-682. [Crossref]

34. Jenkinson M, Bannister P, Brady M, Smith S (2002) Improved optimization for the robust and accurate linear registration and motion correction of brain images. Neuroimage 17: 825-841. [Crossref]

35. Oishi K, Faria A, Mori S (2010) JHU-MNI-ss Atlas. [Crossref]

36. Wang JH, Zuo XN, Gohel S, Milham MP, Biswal BB, et al. (2011) Graph theoretical analysis of functional brain networks: test-retest evaluation on short- and long-term resting-state functional MRI data. PLoS One 6: e21976. [Crossref]

37. Wagner RK, Torgesen JK, Rashotte CA (1999) The comprehensive test of phonologica processing.: Austin, TX: Pro-Ed. [Crossref]

38. Brkanac Z, Chapman NH, Igo RP Jr, Matsushita MM, Nielsen K, et al. (2008) Genome scan of a nonword repetition phenotype in families with dyslexia: evidence for multiple loci. Behav Genet 38: 462-475. [Crossref]

39. Richards TL, Aylward EH, Field KM, Grimme AC, Raskind W, et al. (2006) Converging evidence for triple word form theory in children with dyslexia. Dev Neuropsychol 30: 547-589. [Crossref]

40. Mather N, Roberts R, Hammill D, Allen E (2008) Test of Orthographic Competence (TOC). Austin: Pro-Ed. [Crossref]

41. Treiman R, Kessler B (2014) How children learn to write words: New York: Oxford University Press. [Crossref]

42. Mather N, Hammill D, Allen E, Roberts R (2004) Test of Silent Word Reading Fluency TOSWRF. Austin: Pro-Ed.

43. Rubenstein K, Matsushita M, Berninger VW, Raskind WH, Wijsman EM (2011) Genome scan for spelling deficits: effects of verbal IQ on models of transmission and trait gene localization. Behav Genet 41: 31-42. [Crossref]

44. Nagy W, Berninger V, Abbott R (2006) Contributions of morphology beyond phonology to literacy outcomes of upper elementary and middle school students. Journal of Educational Psychology 98: 134-147. [Crossref]

45. Semel E, Wiig EH, Secord WA (2003) Clinical Evaluations of Language Fundamentals ( th $^{\text {th }}$ Edition). Speech \& Language. [Crossref]

46. Wolf M, Denckla M (2005) RAN/RAS Rapid Automatized Naming and Rapid Alternating Stimulus Tests.: Austin, TX: Pro-Ed. [Crossref]

47. Baddeley A, Gathercole S, Papagno C (1998) The phonological loop as a language learning device. Psychol Rev 105: 158-173. [Crossref]

48. Rubenstein KB, Raskind WH, Berninger VW, Matsushita MM, Wijsman EM (2014) Genome scan for cognitive trait loci of dyslexia: Rapid naming and rapid switching of letters, numbers, and colors. Am J Med Genet B Neuropsychiatr Genet 165B: 345-356. [Crossref]

49. Delis D, Kaplan E, Kramer J (2001) Delis-Kaplan Executive Function System. Clinical Psychology. [Crossref]

50. Tukey JW (1980) We need both exploratory and confirmatory. The American Statistician 34: 23-25. [Crossref]

51. Posner MI, Rothbart MK (2007) Research on attention networks as a model for the integration of psychological science. Annu Rev Psychol 58: 1-23. [Crossref]

52. Cahill L, Tiberius C, Herring J (2013) PolyOrth: Orthography, phonology, and morphology in the inheritance lexicons. Written Language and Literacy 16: 146-185. [Crossref] 
Richards TL (2017) Self-government of complex reading and writing brains informed by cingulo-opercular network for adaptive control and working memory components for language learning

53. Curran S, Mill J, Sham P, Rijsdijk F, Marusic K, et al. (2001) QTL association analysis of the DRD4 exon 3 VNTR polymorphism in a population sample of children screened with a parent rating scale for ADHD symptoms. Am J Med Genet 105: 387-393. [Crossref]

54. Farone S, Mick C (2010) Molecular genetics of attention deficit hyperactivity disorder. Psychiatric Clinics of North America 33: 159-180. [Crossref]

55. Turic D, Swanson J, Sonuga-Barke E (2010) DRD4 and DAT1 in ADHD: Functional neurobiology to pharmacogenetics. Pharmgenomics Pers Med 3: 61-78. [Crossref]
56. Pennington BF (2005) Toward a new neuropsychological model of attention-deficit/ hyperactivity disorder: subtypes and multiple deficits. Biol Psychiatry 57: 1221-1223. [Crossref]

57. Abbott R, Raskind W, Matsushita M, Richards T, Price N, et al. (2017) Dysgraphia dyslexia, and OWL LD during middle childhood and early adolescence: Evidence for genetic effects on hallmark phenotypes. Biomarkers and Genes 1: 1-10.

58. Hood L, Price ND (2014) Demystifying disease, democratizing health care. Sci Transl Med 6: 225ed5. [Crossref].

Copyright: (C2017 Richards TL. This is an open-access article distributed under the terms of the Creative Commons Attribution License, which permits unrestricted use, distribution, and reproduction in any medium, provided the original author and source are credited. 